\title{
Hemorragia intraventricular del prematuro e hidrocefalia post-hemorrágica. Propuesta de un protocolo de manejo basado en la derivación ventrículo-peritoneal
}

\section{precoz}

B. Ros-López; A.M. Jaramillo-Dallimonti; L.S. De Miguel-Pueyo; S. Rodríguez-Barceló; M. Domínguez-Páez; G. Ibáñez-Botella; B. Márquez-Márquez y M.A. Arráez-Sánchez

Servicio de Neurocirugía. Hospital Regional Universitario Carlos Haya. Málaga.

Resumen

Introducción. Aproximadamente el $\mathbf{5 0 \%}$ de los pacientes pretérminos diagnosticados de hemorragia intraventricular desarrollará una hidrocefalia posthemorrágica. La derivación ventrículo-peritoneal de LCR puede constituir en muchos casos la única opción de tratamiento definitivo, aunque se han descrito elevados porcentajes de complicaciones infecciosas o por obstrucción, existiendo además debate sobre cual es el momento más adecuado para la intervención.

Objetivo. Presentar un protocolo de diagnóstico y tratamiento de la hidrocefalia post-hemorrágica del prematuro y describir nuestra experiencia inicial con su aplicación en el Hospital Materno-Infantil Carlos Haya de Málaga.

Material y métodos. Un total de 21 pacientes con diagnóstico de hidrocefalia post-hemorrágica del prematuro fueron intervenidos mediante derivación ventrículo-peritoneal entre enero de 2003 y diciembre de 2006 de acuerdo al protocolo de diagnóstico y tratamiento elaborado en nuestro centro. En todos los casos se trataba de hidrocefalias grado III o IV de Papile, con dilataciones ventriculares graves (índice tálamo-caudado superior a $1.5 \mathrm{~cm}$ ) y de presentación subaguda o crónica. Se emplearon válvulas de presión media y catéteres con impregnación antibiótica. Se consideró 1500 gr como el peso mínimo necesario para la intervención. Se valoró la aparición de complicaciones postquirúrgicas y la situación funcional de los pacientes en el seguimiento ambulatorio clasificándola en cuatro grados (excelente o grado 1, buena o grado 2, regular o grado 3 y mala o grado 4) de acuerdo con la presencia de focalidad, la relación con el entorno y presencia o no de crisis comiciales.

Resultados. Las complicaciones más frecuentes fueron: cráneo escafocefálico en 5 pacientes, colección subgaleal persistente en 2 casos, ventrículo en hendidura sintomático en 2 casos, dehiscencia de herida quirúrgica en 1 caso con posterior infección valvular.

Recibido: 17-01-08. Aceptado: 18-06-08
Un paciente presentó una infección micótica sistémica con compromiso meníngeo no diagnosticado previo a la derivación. Se requirió recambio del sistema de derivación en 7 pacientes (14 reintervenciones); en 2 de estos casos se realizó septostomía endoscópica por hidrocefalia tabicada (junto con ventriculostomía de III ventrículo fallida) y en un tercer caso se realizó una ventriculostomía endoscópica y retirada valvular tras un episodio de disfunción valvular, con fallo diferido de la ventriculostomía. En cuanto a resultados funcionales 9 pacientes se clasificaron como grado 1,5 pacientes como grado 2, 3 pacientes como grado 3 y 4 pacientes como grado 4 , con un $67 \%$ de resultados buenos o excelentes.

Conclusiones. Proponemos un protocolo para el diagnóstico y tratamiento de la hidrocefalia posthemorrágica del prematuro, de aplicación en nuestro Servicio desde Enero de 2003. En nuestra experiencia es posible derivar pacientes a partir de 1500 gr de peso con baja morbilidad. La protocolización puede ayudarnos a reducir complicaciones y a mejorar el pronóstico funcional de estos pacientes.

PALABRAS CLAVE: Hidrocefalia. Hemorragia intraventricular. Prematuro. Derivación ventrículo-peritoneal.

Ventricular haemorrhage in preterm neonates and posthemorrhagic hydrocephalus. Proposal of a management protocol based on early ventriculo-peritoneal shunt

\section{Summary}

Introduction. About $50 \%$ of the preterm neonates with a ventricular haemorrhage will develop post-

Abreviaturas. DVE: drenaje ventricular externo. DVP: derivación ventrículo-peritoneal. HIV: hemorragia intraventricular. HMG: hemorragia de la matriz germinal. HPH: hidrocefalia posthemorrágica. HPHP: hidrocefalia posthemorrágica del prematuro. HSE: hemorragia subependimaria. MG: matriz germinal. PC: perímetro cefálico. VPM: ventriculostomía premamilar (EVT: endoscopic third ventriculostomy). 
haemorrhagic hydrocephalus. Medical treatment is not effective neither safe, does not reduce shunt's dependence and therefore can not be recommended; early and repetitive ventricular or lumbar punctures and the use of intraventricular fibrynolitic treatment have showed no effect on reducing patient's disability, shunt's necessity or mortality of these patients and furthermore, they can have several and important side effects. The ventriculo-peritoneal shunt can be in many cases the only option for definitive treatment, despite well-known infective and obstructive complications and there is an ongoing debate about the ideal moment for the intervention.

Objective. To present a diagnostic and treatment protocol for post-haemorrhagic hydrocephalus of the preterm and describe our initial experience with its application on the Paediatric Neurosurgical Department at the Hospital Materno-Infantil Carlos Haya of Málaga.

Materials and methods. A total of 21 patients with diagnosis of preterm post-haemorrhagic hydrocephalus were surgically treated at our hospital with ventriculoperitoneal shunt between January 2003 and September 2006 following the designed protocol. All the cases were Papile's grade III or IV with severe ventricular dilation (Thalamus-Caudate index over $1.5 \mathrm{~cm}$ ) and subacute or chronic presentation. We used medium pressure valves and antibiotic impregnated catheters. We considered $1500 \mathrm{~g}$ as the minimum weight permitted for the intervention. We report the early and late postoperative complications and the patients functional state at the ambulatory follow up classifying them in $\mathbf{4}$ grades (Excellent or Grade 1; Good or Grade 2; Regular or Grade 3; Poor or Grade 4) according to the presence of neurological focal signs, relation with the surrounding environment, response to stimuli and presence of seizures.

Results. The most frequent complications were escaphocephalic cranium in 5 patients, persistent subgaleal collections in 2 patients, symptomatic slit ventricles in 2 patients and surgical wound dehiscence with shunt infection in 1 patient. One patient presented a systemic fungical infection with non-diagnosed meningeal compromise previous to the shunt. 7 patients required shunt replacement (14 procedures); in 2 cases of tabicated hydrocephalus an endoscopical septostomy (associated with an ETV that did not function) was done, and in a third case ETV and shunt removal was performed after shunt malfunction, with delayed failure of ETV. For the functional results 9 patients were classified as Grade 1, 5 patients as Grade 2, 3 patients as Grade 3 and 4 patients as Grade 4 . This means a $67 \%$ of good or excellent results.
Conclusions. We propose a diagnostic and treatment protocol for preterm neonates with haemorrhagic hydrocephalus that we have been using since 2003 at our department. In our experience it is possible to shunt patients starting at $1500 \mathrm{~g}$ with low morbidity. The use of protocols can help in reducing complications and improving functional results in these patients.

KEY WORDS: Hydrocephalus. Intraventricular haemorrhage. Preterm neonates. Ventricular peritoneal shunt.

\section{Introducción}

La revisión de la literatura respecto al manejo de la hidrocefalia post-hemorrágica del prematuro nos muestra que el tratamiento médico no es efectivo ni seguro, no reduce la dependencia de la válvula $\mathrm{y}$, por lo tanto, no puede ser recomendado ${ }^{17,18}$. Opciones ampliamente utilizadas durante años como las punciones lumbares o ventriculares precoces y repetidas o el uso de fibrinolisis intraventricular se han mostrado ineficaces a la hora de reducir la discapacidad, la necesidad de derivación licuoral o la mortalidad en estos pacientes, y además pueden tener efectos adversos importantes ${ }^{15,16,17,18}$. En ocasiones, como única alternativa, el neurocirujano tiene que recurrir a la derivación de LCR en pacientes considerados clásicamente malos candidatos a cirugía por el alto índice de complicaciones esperables, no existiendo ni siquiera acuerdo en cuanto al peso mínimo necesario para la intervención ${ }^{2,3,12,14}$. En la práctica habitual el interés del neurocirujano se centra en el seguimiento de aspectos relacionados con la cirugía, con poco énfasis en el pronóstico funcional. En nuestra opinión la protocolización conduciría a la mejora en la atención inicial y el seguimiento ambulatorio de estos pacientes.

\section{Materiales y métodos}

Entre enero de 2003 y diciembre de 2006 fueron intervenidos quirúrgicamente un total de 21 pacientes con diagnóstico de HPH del prematuro en la Sección de Neurocirugía Infantil del Servicio de Neurocirugía del Hospital Regional Universitario Carlos Haya de Málaga (Tablas 1 y 2). Se trataba en todos los casos de hidrocefalias grado III o IV de Papile, con dilataciones ventriculares graves (índice tálamo-caudado superior a $1.5 \mathrm{~cm}$ ) y presentación subaguda o crónica. Se valoraron complicaciones postquirúrgicas precoces y tardías (Tabla 1) y la situación funcional de los pacientes en el seguimiento ambulatorio, empleando una escala de desarrollo propio (Tabla 3). Se aplicó el protocolo de diagnóstico y tratamiento diseñado en nuestro Servicio que se expone a continuación y que representamos esquemáticamente en el Anexo 1. 


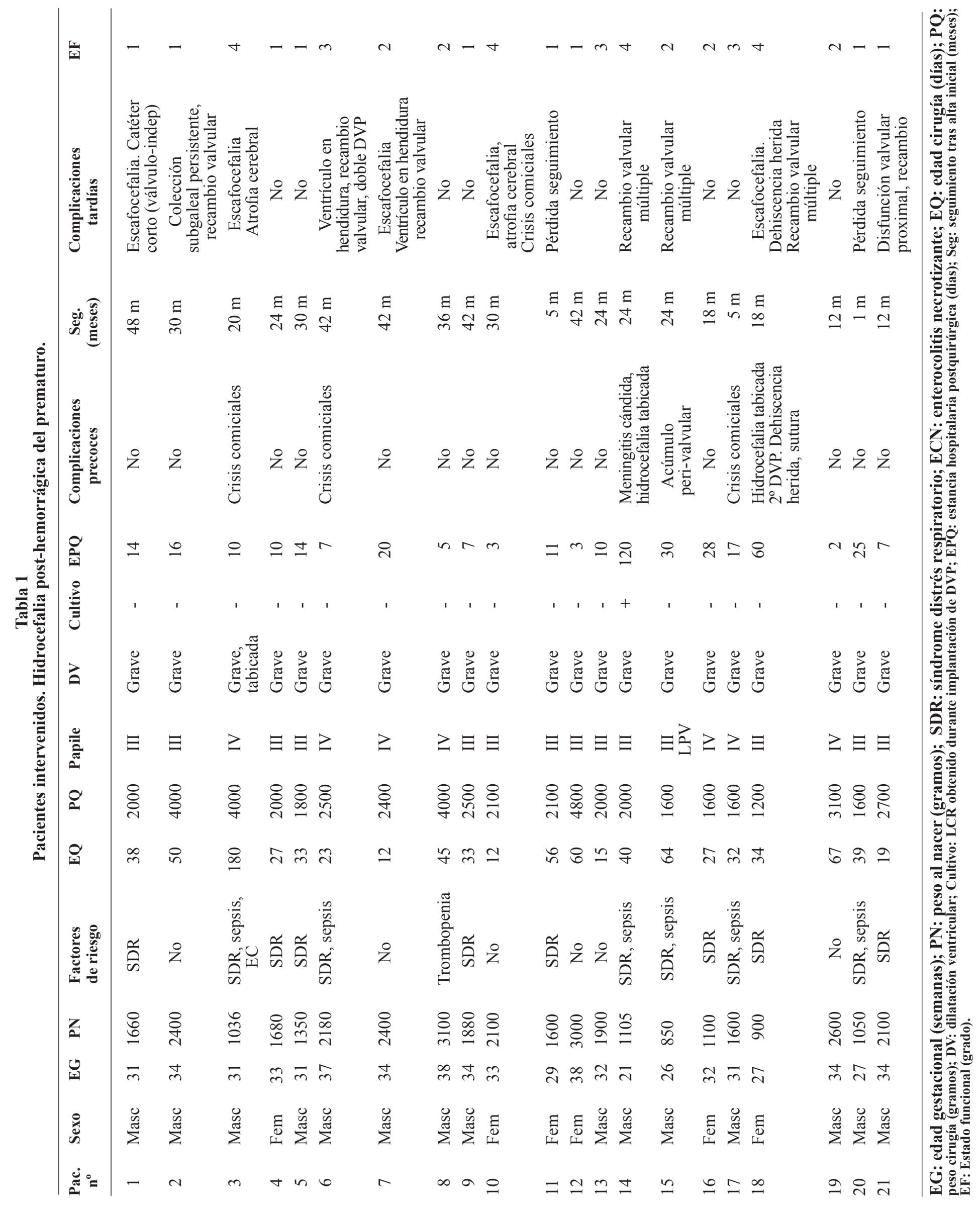


Tabla 2

Datos globales de la serie (21 pacientes)

\begin{tabular}{|l|c|}
\hline Características & Detalles \\
\hline EG media (rango) & 31.76 semanas $(21-38)$ \\
PN medio & $1790 \mathrm{gr}(850-3100)$ \\
Ratio sexo v/m & $14 / 7$ \\
Factores riesgo: & $14 / 21 \mathrm{pac}$ \\
$\quad$ SDR & $6 / 21 \mathrm{pac}$ \\
Sepsis & $1 / 21 \mathrm{pac}$ \\
Trombopenia & \\
Papile & 14 pac \\
III & $7 \mathrm{pac}$ \\
IV & 43.14 días $(12-180)$ \\
EQ media & $2450 \mathrm{gr}(1200-4800)$ \\
PQ medio & 19.95 días $(2-120)$ \\
EPQ media & 25.19 meses $(1-42)$ \\
Seguimiento & \\
Complicaciones & 5 pac $(23.8 \%)$ \\
Escafocefalia & 12 pac $(9.52 \%)$ \\
Colección subgalea & 2 pac $(9.52 \%)$ \\
Colapso ventr. Sintom & 1 pac $(4.76 \%)$ \\
Dehiscencia herida/infecc & 1 pac $(4.75 \%)$ \\
Infec. meníngea previa & 2 pac $(9.52 \%)$ \\
Pérdida seguimiento & 7 pac, 14 reinterv \\
No reintervenciones & 15 pac \\
No reinterv. obstr. proxima & \\
Situación funcional & 9 pac $(42.85 \%)$ \\
Excelente: grado 1 & 5 pac $(23.28 \%)$ \\
Buena: grado 2 & 3 pac $(14.28 \%)$ \\
Regular: grado 3 & 4 pac $(19 \%)$ \\
Mala: grado 4 & a \\
\hline
\end{tabular}

EG: edad gestacional; PN: peso al nacer; SDR: síndrome del distrés respiratorio; EQ: edad en el momento de la cirugía; PQ: peso en el momento de la cirugía; EPQ: estancia hospitalaria postquirúrgica

Protocolo de diagnóstico y tratamiento en HIDROCEFALIA POSTHEMORRÁGICA DEL PREMATURO (HPHP).

Basado en los siguientes principios:

1. Diagnóstico precoz de hemorragia subependimaria (HSE) o de matriz germinal (HMG) mediante "screening" (ECO transfontanelar) en población de riesgo.
Tabla 3

Clasificación funcional

\begin{tabular}{|c|c|c|c|c|}
\hline & $\begin{array}{c}\text { Excelente } \\
\text { (1) }\end{array}$ & $\begin{array}{c}\text { Buena } \\
\text { (2) }\end{array}$ & $\begin{array}{c}\text { Regular } \\
\text { (3) }\end{array}$ & $\begin{array}{l}\text { Mala } \\
\text { (4) }\end{array}$ \\
\hline $\begin{array}{l}\text { Ausencia } \\
\text { de } \\
\text { focalidad }\end{array}$ & Sí & No & No & No \\
\hline $\begin{array}{l}\text { Interacción/ } \\
\text { Juegos }\end{array}$ & Sí & Sí & No & No \\
\hline $\begin{array}{l}\text { Respuesta a } \\
\text { estímulos }\end{array}$ & Sí & Sí & Sí & No \\
\hline
\end{tabular}

*La presencia de crisis comiciales suma un grado a 1, 2 ó 3

2. Seguimiento estricto de pacientes con HSE diagnosticada para detección precoz de HPH. En grados I y II de Papile el seguimiento corre a cargo de Neonatología mediante controles periódicos de perímetro cefálico (PC) y ecografías seriadas. Neurocirugía interviene directamente en el seguimiento de grados III y IV.

3. Selección de pacientes candidatos a tratamiento quirúrgico mediante criterios clínicos y radiológicos. Un elemento esencial es el carácter progresivo de la macrocefalia y de la ventriculomegalia, teniendo en cuenta que un porcentaje de pacientes desarrollará hidrocefalias "ex-vacuo" o hidrocefalias transitorias, no precisando derivación. En la mayoría de los casos la presentación clínica será subaguda o crónica por lo que la indicación consistirá en derivación ventrículoperitoneal primaria. No realizamos rutinariamente estudio de LCR preoperatorio salvo sospecha clínica de infección meníngea.

4. Reducción al mínimo del número de punciones lumbares o ventriculares. Consideramos indicación de punción evacuadora:

a. Para estudio de LCR si existe sospecha clínica de infección meníngea.

b. Como medida descompresiva de "emergencia" en hidrocefalias de presentación aguda.

c. Como medida "transitoria" mientras se establece la viabilidad del neonato.

d. Como medida de "espera" mientras se alcanza el peso o situación sistémica considerada necesaria para la intervención. Se realizan el mínimo número imprescindible de punciones para el control de la hipertensión intracraneal. Habitualmente es suficiente con una o dos punciones fontanelares por semana con evacuación de 20-30 cc de LCR.

5. Indicación de drenaje ventricular externo (DVE) exclusivamente en los siguientes supuestos:

a. Infección meníngea confirmada, para tratamiento combinado intravenoso e intratecal y control 


\section{Anexo 1. Algoritmo diagnóstico-terapéutico en HPHP}

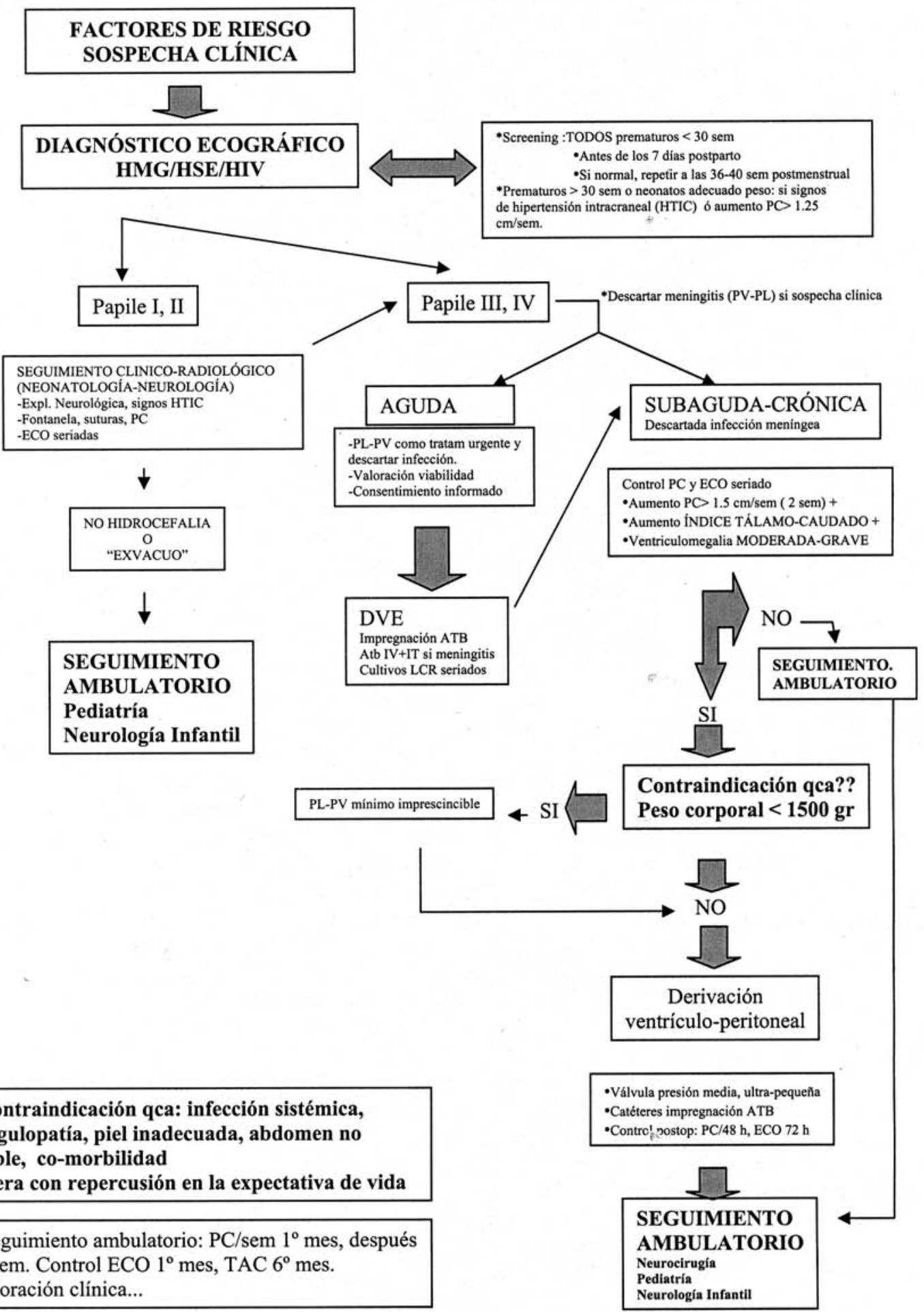


seriado de LCR.

b. Presentación aguda, con necesidad de punciones evacuadoras repetidas de forma prolongada para control de hipertensión intracraneal, en pacientes considerados viables y tras obtención de consentimiento informado.

6. Tratamiento quirúrgico mediante DERIVACIÓN VENTRÍCULO-PERITONEAL. Aplicación de protocolo de "alto riesgo de infección" con utilización de catéteres con impregnación antibiótica. Empleamos válvulas de presión media, "ultra-pequeñas". Consideramos que existe indicación quirúrgica cuando:

a. Existe un aumento progresivo y patológico de PC $>1.5 \mathrm{~cm} /$ semana durante 2 semanas junto con aumento de índices ventriculares en al menos dos ecografías seriadas y siempre y cuando el índice tálamo-caudado sea superior a $1 \mathrm{~cm}$ (ventriculomegalia moderada o grave).

b. No existe contraindicación quirúrgica. Consideramos contraindicación para derivación de LCR:

- Infección sistémica

- Coagulopatía

- Piel "inadecuada"

- Co-morbilidad severa con repercusión en la expectativa de vida

- Abdomen "no viable" (enterocolitis necrotizante...)

c. El peso del paciente se aproxima o supera los $1500 \mathrm{gr}$

d. No contraindicamos la cirugía en función de la concentración de proteínas, número de hematíes o color del LCR.

7. Seguimiento postoperatorio. Control de PC cada 48 horas, control ecográfico a las 72 horas aproximadamente y vigilancia de heridas y zonas quirúrgicas (evitando apoyo sobre zona valvular). Retirada de sutura a los 8 días post-cirugía. Tras el alta control de PC semanal (pediatra) con ecografía de control al mes y de TAC al $6^{\circ}$ mes (después controles según situación clínica). En la revisión ambulatoria se interroga sobre la posible aparición de complicaciones postquirúrgicas y se clasifica la situación funcional de los pacientes en cuatro grados (excelente o grado 1, buena o grado 2, regular o grado 3 y mala o grado 4) (Tabla 3). Esta sencilla escala fue desarrollada teniendo en cuenta los elementos esenciales examinados por el neurocirujano en Consulta Externa (exploración, informes de especialistas, anamnesis dirigida a los padres) con objeto de estandarizar y facilitar el control ambulatorio en nuestro Servicio:

a. Ausencia/presencia de focalidad neurológica en la exploración (paresia espástica). Se buscan anomalías o asimetrías de tono o fuerza durante la movilización pasiva de extremidades. b. Interacción con el entorno: se valora desde la aparición de sonrisa social en los primeros meses de vida hasta la participación en juegos (adecuados para la edad del paciente).

c. Respuesta a estímulos visuales, auditivos o táctiles: dirige cabeza o mirada al estímulo, coge objetos.

d. Presencia o historia de crisis comiciales que precisan medicación: se ha relacionado con las secuelas de la HIV / HPH, siendo considerada factor de mal pronóstico funcional ${ }^{4}$.

En función de estos parámetros hablaríamos de situación funcional:

a. Excelente o grado 1: no existe diferencia respecto al patrón de desarrollo psicomotriz de un niño sano. Ausencia de focalidad neurológica. Interacción adecuada con el entorno. Responde a estímulos.

b. Buena o grado 2: existe focalidad neurológica (paresia espástica) que precisa o ha precisado tratamiento fisioterápico. Interacción con el entorno y respuesta a estímulos. Incluye grados 1 con crisis comiciales.

c. Regular o grado 3: focalidad neurológica, no interacción con el entorno aunque sí presenta respuesta a estímulos. Incluye grados 2 con crisis comiciales.

d. Mala o grado 4: focalidad neurológica, no interacción con el entorno, no respuesta a estímulos. Incluye grados 3 con crisis comiciales.

\section{Resultados}

Las complicaciones más frecuentes fueron (ver Tabla 1):

a) Cráneo escafocefálico (con repercusión estética): se detectó en 5 pacientes $\left(\mathrm{n}^{\mathrm{o}} 1,3,7,10\right.$ y 18), asociado a colapso ventricular radiológico y sintomático en uno de ellos $\left(n^{\circ} 7\right)$. En tres pacientes ( $n^{\circ} 3$ y 10) existe atrofia cerebral marcada con mala situación funcional y en uno $\left(n^{\circ} 1\right)$ se observa el catéter ventricular fuera del sistema ventricular con mínima dilatación del mismo y con situación funcional excelente (considerado independiente del sistema derivativo).

b) Colección subgaleal persistente en 2 casos. En ninguno se produjo fístula externa de LCR por herida quirúrgica ni aparecieron signos clínicos o radiológicos de disfunción valvular. En un caso la presentación fue precoz en el postoperatorio $\left(n^{\circ} 15\right)$ y en otro diferida $\left(n^{\circ} 2\right)$. Motivó el recambio valvular por válvulas programables a muy baja presión, con sobredrenaje transitorio hasta resolución de la colección, para posterior reprogramación a presiones más altas. En el caso $n^{\circ} 15$ la intervención se realizó en el $4^{\circ}$ mes de vida con resultado satisfactorio aunque quedando el catéter proximal alojado en el 
asta temporal; con posterioridad presentó un episodio de disfunción valvular proximal a los 11 meses edad, siendo tratado mediante ventriculostomía premamilar endoscópica y retirada valvular. A pesar del éxito inicial 9 meses después ingresa de nuevo con decaimiento y vómitos y dilatación ventricular significativa en el TAC: la RMN realizada es compatible con fallo diferido de ventriculostomía por lo que se procede a una nueva derivación de LCR.

c) Ventrículos en hendidura sintomáticos. Se observó en 2 casos ( $\mathrm{n}^{\mathrm{o}} 6$ y 7), con episodios de decaimiento y vómitos autolimitados que finalmente desembocan en obstrucción completa del catéter ventricular. En el caso $n^{\circ} 6$ el colapso ventricular fue unilateral con sintomatología intermitente que terminó en obstrucción completa del catéter ventricular -con posterior dilatación bilateral aunque asimétrica del sistema ventricular- y que fue tratada mediante la colocación de nuevo sistema derivativo con válvula programable contralateral y revisión del catéter ventricular previo (actualmente portador de doble sistema derivativo). En el segundo $\left(\mathrm{n}^{\circ} 7\right)$ se produce también obstrucción proximal con dilatación ventricular contralateral a nivel frontal (porencefalia previa): se procedió a recambio valvular por válvula programable, con posterior ascenso progresivo de presión de apertura por clínica de hipertensión intracraneal (colapso ventricular sintomático) hasta 160 $\mathrm{mmH2O}$, con resolución transitoria de los síntomas. $\mathrm{Su}$ reaparición motivó la reintervención, con adición distal al reservorio valvular de un dispositivo antigravitacional y reprogramación de la presión de apertura a 100 $\mathrm{mmH} 2 \mathrm{O}$ (actualmente sin síntomas aunque con colapso ventricular radiológico).

d) Dehiscencia parcial de herida quirúrgica en 1 caso $\left(\mathrm{n}^{\circ}\right.$ 18). La paciente, como excepción al protocolo, fue intervenida con $1100 \mathrm{gr}$ de peso por hidrocefalia subaguda con fontanela a tensión tras tres punciones evacuadoras, implantándose derivación ventrículo-peritoneal con tubo libre, con el objeto de evitar la colocación de un DVE. En el postoperatorio presentó dehiscencia parcial de herida quirúrgica craneal, precisando resutura de la misma. Posteriormente la paciente desarrolla tabicación ventricular por lo que, ya con $1500 \mathrm{gr}$ de peso, se coloca una segunda válvula contralateral (en la Tabla 2 se recogen los datos de la paciente en la primera cirugía). De forma diferida, tras el alta hospitalaria, reaparece la dehiscencia, por lo que se sospecha infección del sistema derivativo. Se procedió a retirada de ambas válvulas y a la realización por vía endoscópica de septostomía múltiple y ventriculostomía premamilar endoscópica, con colocación de drenaje ventricular externo transitorio como medida de seguridad. En el postoperatorio se comprueba la ausencia de funcionamiento de la ventriculostomía, por lo que se procede a la apertura del drenaje externo; los cultivos seriados mostraron contaminación por estafilocócica por lo que se instauró tratamiento antibiótico intravenoso e intratecal hasta su negativización, con posterior colocación de válvula única programable a baja presión. Los controles de RMN muestran reducción del tamaño ventricular asimétrico y IV ventrículo aislado, aunque dada la mejoría funcional de la paciente, se ha tomado una actitud expectante.

e) Un paciente $\left(\mathrm{n}^{\circ} 14\right)$ presentó una infección micótica sistémica con compromiso meníngeo previo a la derivación, no detectada al no realizarse de forma rutinaria estudio preoperatorio de LCR. El LCR obtenido durante la cirugía mostró colonización por cándida, lo que obligó la retirada del sistema valvular, la implantación de un drenaje ventricular externo y tratamiento antifúngico intravenoso e intratecal. La tabicación ventricular subsiguiente fue tratada mediante septostomía endoscópica múltiple y ventriculostomía premamilar. A pesar de un postoperatorio inicialmente satisfactorio, el paciente desarrolló de nuevo hidrocefalia activa de forma diferida ( 1 mes después del tratamiento endoscópico) con colocación de nuevo sistema derivativo. Meses después presentó obstrucción del catéter ventricular (catéter corto), procediéndose a recambio valvular completo.

f) Se llevaron a cabo inicialmente 22 intervenciones quirúrgicas en 21 pacientes (dos en el paciente $n^{\circ} 18$ ). Se requirió posteriormente recambio del sistema de derivación en un total de 7 pacientes (33\%), con 14 reintervenciones (pacientes $\mathrm{n}^{\mathrm{o}} 2,6,7,14,15,18$ y 21 ). En total se revisaron los sistemas derivativos por obstrucción proximal en cinco casos $\left(\mathrm{n}^{\circ} 6,7,14,15\right.$ y 21$)$ : en los pacientes $n^{\circ} 6$ y 7 la obstrucción se relacionó con colapso ventricular; en el $\mathrm{n}^{\circ} 14$ por catéter ventricular corto; en el $\mathrm{n}^{\mathrm{o}} 15$ por malposición de catéter proximal en el asta temporal, con obstrucción del mismo, y en el $\mathrm{n}^{\mathrm{o}} 21$ por obstrucción diferida por material hemático (10 meses después de la derivación).

g) En dos casos ( $n^{\circ} 15$ y 18) se realizaron punciones ventriculares previas a la cirugía, como medida de control transitorio de la presión intracraneal, hasta alcanzar el peso corporal considerado adecuado para la intervención (dos y tres punciones respectivamente).

h) En dos casos $\left(n^{\circ} 11\right.$ y 20) se produjeron pérdidas precoces en el seguimiento postoperatorio, por lo que la valoración funcional puede ser poco significativa. No obstante, en el resto de casos las puntuaciones obtenidas en la primera valoración funcional se mantuvieron constantes a lo largo del seguimiento, por lo que incluimos también las de los dos casos mencionados.

En cuanto a la situación funcional en el seguimiento (ver Tabla 1, donde se recoge la puntuación en la última revisión): 9 pacientes se clasificaron como grado 1, 5 
pacientes se clasificaron como grado 2, 3 pacientes como grado 3 y 4 pacientes como grado 4 . En conjunto los resultados fueron excelentes o buenos en un $67 \%$ de los casos.

\section{Discusión}

Aproximadamente el 50\% de los pacientes prematuros diagnosticados de HIV desarrollará una hidrocefalia posthemorrágica ${ }^{2,5}$. La población de riesgo se encuentra en aumento en nuestro medio en relación con el desarrollo de técnicas de reproducción asistida y aumento de partos múltiples, así como por la mejoría en la expectativa vital de estos pacientes que acompaña a la especialización de las Unidades de Cuidados Intensivos neonatales. Los neurocirujanos reciben cada vez más consultas que requieren una decisión terapéutica, debido también a la mayor y mejor aplicación de técnicas de diagnóstico por imagen y a la falta de éxito de estrategias de tratamiento médico. El neurocirujano debe elegir en cada caso la mejor opción terapéutica, es decir, aquella que permita el control de la hidrocefalia (de la hipertensión intracraneal y de la ventriculomegalia) de forma precoz, prolongada, si es posible permanente, y con el menor porcentaje de complicaciones posible. Además ha de pensar en términos de reducción de gasto sanitario, de estancia hospitalaria y sobre todo de pronóstico funcional a largo plazo de los pacientes. De un manejo médico-quirúrgico adecuado en estas primeras etapas dependerá en parte la expectativa futura de calidad de vida.

El tratamiento precoz de la hidrocefalia supondría un mejor pronóstico funcional, ya que el proceso de maduración neuronal se vería alterado por la duración de la dilatación ventricular en el periodo intraútero ${ }^{11}$; ésta, aún en ausencia de signos manifiestos de hipertensión intracraneal, afectaría al proceso de mielinización, efecto que sería reversible con un tratamiento precoz $^{7}$. Sin embargo parece claro que las complicaciones quirúrgicas (dehiscencia de heridas, decúbitos, fístula de LCR, infección, obstrucción por restos hemáticos) se reducen con un tratamiento diferido ${ }^{5,14}$. De las distintas alternativas diseñadas para evitar o retrasar la derivación de LCR no hay ninguna exenta de complicaciones. Las punciones ventriculares precoces y repetidas, los diuréticos y el uso de fibrinolisis intraventricular a través de drenajes externos se han mostrado ineficaces a la hora de reducir la discapacidad, la dependencia de la derivación licuoral definitiva o la mortalidad ${ }^{15,16,17,18}$. El uso de reservorios de Ommaya para punciones repetidas se ha extendido por el menor número de problemas en relación con los drenajes externos o la derivación ventrículo-subgaleal: permiten el control de la hidrocefalia durante semanas, aunque se han descrito como complicaciones dehiscencia de la herida (12\%), fístula de LCR (15\%) y obstrucciones del sistema $(20 \%)^{7}$. Esta técnica ha sido empleada como parte de un protocolo de tratamiento para evitar la derivación de LCR definitiva o para retrasar el tratamiento con DVP o VPM ${ }^{12}$. La VPM endoscópica ha mostrado un bajo porcentaje de éxito en el tratamiento primario de la $\mathrm{HPH}$, aunque sin embargo constituye una alternativa a la revisión valvular tras disfunción e incluso podría ser el tratamiento de elección $^{12}$. Recientemente se propuso una nueva modalidad de tratamiento que combinaba drenaje, irrigación y terapia fibrinolítica (conocida como DRIFT, acrónimo de DRainage, Irrigation and Fibrinolytic Therapy): los autores recomendaron su aplicación precoz para mejorar resultados, publicando en estudios preliminares un porcentaje de válvulo-dependencia de sólo el $24 \%$ con una tasa de infección del 8\% y de hemorragias secundarias del 8\%. Tendría como objetivo el "lavado" de sangre intraventricular y de sus subproductos, tales como citoquinas proinflamatorias, responsables de perpetuar la hidrocefalia al causar depósito de proteínas de matriz extracelular, con aracnoiditis basal y de granulaciones aracnoideas ${ }^{19}$. Sin embargo se ha descrito que tales citoquinas, como el transforming growing factor $\beta$ (TGF $\beta$ ), se expresan tras una lesión cerebral de cualquier origen (p.e. traumática o isquémica) e incluso su presencia podría ser la consecuencia y no la causa de la dilatación ventricular ${ }^{4,6}$. Los resultados de un ensayo clínico randomizado que comparaba los resultados del DRIFT frente a punciones repetidas de reservorio valvular han mostrado un porcentaje similar de válvula-dependencia pero con complicaciones hemorrágicas hasta en el 35\% de los pacientes del primer grupo, por lo que esta modalidad de tratamiento no puede ser recomendada hoy en día ${ }^{20}$.

Después de repasar las distintas alternativas de tratamiento de la HPH del prematuro se comprueba que, en el momento actual, no existe ninguna que sea ideal o que pueda ser considerada de elección. Frecuentemente, como mejor alternativa, el neurocirujano tiene que recurrir a la derivación de LCR en pacientes considerados clásicamente malos candidatos a cirugía por el alto índice de complicaciones esperables, no existiendo ni siquiera acuerdo en cuanto al peso mínimo necesario para la intervención ${ }^{2,3,12,14}$. El desarrollo de sistemas derivativos de menor tamaño y quizá el uso de catéteres con impregnación antibiótica han contribuido a disminuir la morbi-mortalidad del procedimiento ${ }^{13}$. Además del bajo peso de los neonatos, las características del LCR podrían influir en el desarrollo de complicaciones tras un procedimiento derivativo: mientras que la proteinorraquia no afectaría a la función valvular, la celularidad -especialmente la hematorraquia- sí que incrementaría el riesgo de disfunción del sistema․ Para paliar este efecto se ha propuesto un periodo de espera que permita el "aclaramiento" del LCR, disminuyendo así la tasa de obstrucción por restos de sangre ${ }^{14}$. En distintos centros se han desarrollado protocolos de derivación ventrículoperitoneal en edades cada vez más tempranas, con buenos resultados incluso a partir de los 1400 gr de peso $^{2}$. Esta 
actitud permitiría, a diferencia de otras modalidades de tratamiento, el control continuo y prolongado de la presión intracraneal y la reducción de la ventriculomegalia en un periodo de la vida en el que no se ha completado el desarrollo de la corteza cerebral ni de la sustancia blanca mielinizada, mejorando las expectativas de desarrollo psicomotor a largo plazo ${ }^{7,8}$.

En la literatura han sido relacionados con el pronóstico funcional de los pretérminos las características del paciente (grado de prematuridad, edad gestacional, peso corporal, parto múltiple, género masculino, etc), complicaciones médicas (síndrome del distrés respiratorio, neumotórax, duración de la ventilación, sepsis, enterocolitis necrotizante...) y factores demográficos maternos (educacionales). Es decir, el simple hecho de la prematuridad implica riesgo de retraso psicomotor e incluso de parálisis cerebral en niños con ecografías normales. Hasta el $30 \%$ de pacientes nacidos con extremo bajo peso y con estudios ecográficos normales tendrían algún grado de retraso psicomotor medido a 18-22 meses. Además, las anomalías ecográficas actúan como predictores de déficit cognitivo o parálisis cerebral $^{9}$. En nuestros pacientes además de la prematuridad y de las anomalías de imagen habría que tener en cuenta las secuelas derivadas de la propia hidrocefalia y de las complicaciones de su tratamiento. De las escalas empleadas en la valoración funcional en prematuros probablemente sea la de Bayley (Bayley Scale of Infant Developmen [BSID]), en sus versiones BSID I (1969) y II (1993) las de mayor difusión. No obstante la gran complejidad de los estudios neuropsicológicos hacen difícil su uso a estas edades en la práctica diaria ${ }^{3,9,10}$, siendo sólo de aplicación por parte de neuropsicólogos o "psicometristas" expertos. Aunque el uso de escalas validadas por equipos multidisciplinarios (neuropediatras, psicólogos infantiles y neurocirujanos) debe ser el objetivo final en la valoración de estos pacientes, pensamos que el neurocirujano no debe renunciar a tener una información básica sobre la evolución en el ámbito funcional en los primeros meses tras el alta hospitalaria, evitando un periodo "en blanco" en el seguimiento. En este sentido hemos desarrollado una clasificación funcional elemental a partir de las preguntas que el neurocirujano realizaba de forma rutinaria en la consulta externa, de modo que pudiera detectarse, en su caso, una evolución desfavorable entre tanto se realiza una valoración más precisa.

En nuestra serie (Tabla 2) la edad gestacional media fue de 31.76 semanas (rango 21-38). Se incluyen dos pacientes de EG de 38 semanas con pesos al nacer superiores a los 3000 gr por presentar una HPH perinatal de características y manejo idénticos al de los verdaderos prematuros. Los factores de riesgo más frecuentes fueron la aparición de SRD o de sepsis; en la mayoría de los pacientes se detectó además algún grado de persistencia del ductus arteriosus. En todos los casos la ecografía reveló grados III (66\%) o
IV de Papile con graves dilataciones ventriculares (índices ventriculares superiores a $1.5 \mathrm{~cm}$ ). La propia "filosofía" del protocolo de "espera", que requiere verificar el carácter progresivo de la ventriculomegalia y alcanzar un peso o condiciones sistémicas mínimas para la cirugía, es en parte responsable de estos hallazgos. Este "retraso" terapéutico es posible, en la mayoría de los casos, dada la cronicidad o lenta progresión de la hidrocefalia y evita el tratamiento de hidrocefalias transitorias o ventriculomegalias "ex-vacuo" (con predominio de atrofia cerebral por leucoencefalopatía de origen isquémico o hemorrágico) garantizando mejores resultados quirúrgicos al reducir el porcentaje de complicaciones. Pensamos que todo ello es compatible con el carácter "precoz" con el que pretendemos derivar a los pacientes que cumplen los criterios de selección y alcanzan un peso de $1500 \mathrm{gr}$. Este límite, similar al empleado en centros de referencia, viene impuesto por nuestra experiencia respecto a la frecuencia de complicaciones postoperatorias en pacientes de muy bajo peso, complicaciones que entendemos son las que verdaderamente ensombrecen el pronóstico funcional.

\section{Conclusiones}

La derivación ventrículo-peritoneal de LCR puede constituir en muchos casos la mejor opción de tratamiento en hidrocefalia posthemorrágica del prematuro. Proponemos un protocolo para el diagnóstico y tratamiento de esta patología, que se aplica en nuestro Servicio desde Enero de 2003. En nuestra experiencia es posible derivar pacientes a partir de 1500 gr de peso con baja morbilidad. La protocolización puede ayudarnos a reducir complicaciones y a mejorar el pronóstico funcional de estos pacientes.

\section{Bibliografía}

1. Brydon, H.L., Bayston, R., Hayward, R., Harkness, W.: The effect of proteins and blood cells on the flow-pressure characteristics of shunts. Neurosurgery 1996; 38: 498-504.

2. Boop, F.A.: Posthemorragic Hydrocephalus of Preamturity. En: Cinalli G, Maixner MJ, Sainte-Rose C (Eds). Pediatric Hydrocephalus. Milano; Springer-Verlag, 2004; pp. 121-131.

3. Boynton, B.R., Boynton, C.A., Merritt, T.A., Vaucher, Y.E., James, H.E., Bejar, R..F.: Ventriculoperitoneal shunts in low birth weight infants with intracranial hemorrhage: neurodevelopmental outcome. Neurosurgery 1986; 18: 141-145.

4. Cherian, S., Thoresen, M., Silvert, I.A., Whitelaw, A., Love, S.: Transforming growth factor Bs in a rat model of neonatal posthaemorrhage hydrocephalus. Neuropathology and Applied Neurobiology 2004; 30: 585-600.

5. Cinalli, G.: Alternatives to shunting. Childs Nerv Syst 1999; 15: 718-731. 
6. Heep, A., Stoffel-Wagner, B., Soddit, V., Aring, C., Groneck, P., Bartmann, P.: Procollagen I C-propeptide in the cerebrospinal fluid of neonates with posthaemorrhagic hydrocephalus. Arch Dis Child Fetal Neonatal 2002; 86: F34-F36.

7. Hudgins, R.J.: Posthemorrhagic hydrocephalus of infancy. Neurosurg Clin N Am 2001; 12: 743-751.

8. Hunt, R.W., Warfield, S.K., Wang, H., Kean, M., Volpe, J.J., Inder, T.E.: Assesment of the impact of the removal of cerebrospinal fluid on the cerebral tissue volumes by advanced 3D-MRI in posthaemorrhagic hydrocephalus in a preterm infant. J Neurol Neurosurg Psychiatry 2003; 74: 658-660.

9. Laptook, A.R., O'Shea, T.M., Shankaran, S, Bhaskar, B.: NICHD Neonatal Network: Adverse neurodevelopmental outcomes among extremely low birth weight infants with a normal head ultrasound: prevalence and antecedents. Pediatrics 2005; 115: 673-680.

10. Niccols, A., Lachtman, A.: Stability of the Bayley Mental Scale of infant development with high risk infants. $\mathrm{Br}$ J Develop Disab 2002; 48: 3-13.

11. Oi, S., Honda, Y., Hidaka, M., Sato, O., Matsumoto, S.: Intrauterine high resolution magnetic resonance imagin in fetal hydrocephalus and prenatal estimation of postnatal outcomes with "perspective classification". J Neurosurg 1988; 685-694.

12. Peretta, P., Ragazzi, P., Carlino, C.F., Gaglini, P., Cinalli, G.: The role of Ommaya reservoir and endoscopic third ventriculostomy in the management of post-hemorrhagic hydrocephalus of prematurity. Childs Nerv Syst 2007; 23: 765-771.

13. Sciubba, D.M., Stuart, R.M., McGirt, M.J., et al: Effect of antibiotic-impregnated catheters in decreasing incidence of shunt infection in the treatment of hydrocephalus. J Neurosurg (Pediatrics 2) 2005; 103: 131-136.

14. Taylor, A.G., Peter, J.C.: Advantages of delayed VP shunting in posthaemorrgagic hydrocephalus seen in lowweight infants. Childs Nerv Syst 2001; 17: 328-333.

15. Ventriculomegaly Trial Group: Randomised trial of early tapping in neonatal posthaemorrhagic ventricular dilatation. Arch Dis Child 1990; 65: 3-10.

16. Ventriculomegaly Trial Group: Randomised trial of early tapping in neonatal posthaemorrhagic ventricular dilatation: results at 30 months. Arch Dis Child 1994; 70: F129F136.

17. Whitelaw, A.: Intraventricular haemorrhage and posthaemorrhagic hydrocephalus pathogenesis, prevention and future interventions. Semin Neonatol 2001; 6: 135-146.

18. Whitelaw, A., Thoresen, M., Pople, I.: Posthaemorrhagic ventricular dilatation. Arch Dis Child Fetal Neonatal Ed 2002; 86: F72-74.

19. Whitelaw, A., Cherian, S., Thoresen, M., Pople, I: Posthaemorrhagic ventricular dilatation: new mechanism and new treatment. Acta Paediatr Suppl 2004; 444: 11-14.

20. Whitelaw, A., Evans, D., Carter, M., et al.: Randomized Clinical Trial of Prevention of Hydrocephalus After Intraventricular Hemorrhage in Preterm Infants: Brain-Washing versus Tapping Fluid. Pediatrics 2007; vol 119: 1071-1078.

Ros-López, B.; Jaramillo-Dallimonti, A.M.; De MiguelPueyo, L.S.; Rodríguez-Barceló, S.; Domínguez-Páez, M.; Ibáñez-Botella, G.; Márquez-Márquez, B; Arráez- Sánchez, M.A.: Hemorragia intraventricular del prematuro e hidrocefalia post-hemorrágica. Propuesta de un protocolo de manejo basado en la derivación ventrículo-peritoneal precoz. Neurocirugía 2009; 20: 15-24.

Correspondencia postal: Servicio de Neurocirugía. Hospital Regional Universitario Carlos Haya. Avda Carlos Haya, s/n. 9010. Málaga. 